\title{
A coherent gradient sensor for crack tip deformation measurements: analysis and experimental results
}

\author{
HAREESH V. TIPPUR, ${ }^{1}$ SRIDHAR KRISHNASWAMY ${ }^{2}$ and ARES J. ROSAKIS \\ Graduate Aeronautical Laboratories, California Institute of Technology, Pasadena, California 91125, USA; \\ present addresses: ${ }^{1}$ Department of Mechanical Engineering, Auburn University, $A L, U S A ;{ }^{2}$ Department of \\ Mechanical Engineering, Northwestern University, IL, USA
}

Received 15 February 1989; accepted in revised form 2 November 1989

\begin{abstract}
A first order diffraction analysis of an optical interferometer, Coherent Gradient Sensor (CGS), for measuring surface gradients is presented. Its applicability in the field of fracture mechanics is demonstrated by quantitatively measuring the gradients of out-of-plane displacements around a crack tip in a three point bent fracture specimen under static loading. This method has potential for the study of deformation fields near a quasi-statically or dynamically growing crack.
\end{abstract}

\section{Introduction}

Presently, several optical methods are being used for measuring elastic or plastic crack tip deformations under quasi-static or dynamic loading conditions. Among the commonly used techniques, photoelasticity [1] measures principal stress differences while moire methods and interferometry [2-4] map in-plane or out-of-plane displacements. In the method of caustics, non-uniform displacement gradients due to crack tip deformations result in the formation of a shadow spot $[5,6]$. Subsequent interpretation of these optical measurements through an assumed asymptotic field description enables one to evaluate the so-called stress intensity factor (SIF) $K_{\mathrm{I}}$ or the $J$-integral which are widely used in characterizing fracture behaviour of materials.

In most of the above methods [1-4], one often encounters the difficult question of whether the chosen experimental technique provides adequate control over the sensitivity of measurement. This becomes relevant because of the wide range of magnitudes of deformation that may occur near a crack tip. Typically, interferometric methods are preferred for the measurement of elastic deformations while for larger deformations the resulting fringe density often overwhelms the recording capabilities. As a result, geometric moiré methods are used when large deformations exist. However, in fracture studies one often needs a method which can satisfactorily perform in both regimes.

In this paper, we propose an optical interferometer - coherent gradient sensor (CGS) to measure in-plane gradients of out-of-plane surface displacements around a crack tip. The method produces high contrast fringes and provides some degree of control on the sensitivity of measurement during quasi-static experiments. In addition, it involves a simple optical set-up and, when compared to other interferometric techniques, it is relatively insensitive to vibrations which makes it a potential candidate for dynamic crack growth applications. Finally, the insensitivity of this method to rigid body motions is highly attractive for solid mechanics applications. 
CGS employs the basic principles of the so-called "moiré deflectometry" used widely in a variety of problems by Kafri and his associates [7, 8]. However, CGS takes advantage of coherent optics by using wave front division (by means of high density Ronchi rulings) and their subsequent interference. By incorporating an online spatial filtering procedure, one could not only realize high contrast fringes in real time, but also use high density diffraction gratings for the purpose.

In the following, we present a diffraction analysis of the method and experimental evidence to demonstrate its applicability for elastic and elasto-plastic crack tip deformation studies.

\section{Experimental method}

\subsection{Experimental set-up}

In Fig. 1 the schematic of the experimental set-up is shown. It consists of a specularly reflective fracture specimen illuminated by a collimated bundle of coherent laser light. Normal incidence is achieved using a beam splitter. The reflected object wave is incident on a pair of high density Ronchi rulings, $G_{1}$ and $G_{2}$, separated by a distance $\Delta$. The principal direction of the rulings is coincident with either the $x_{1}$ - or $x_{2}$-coordinate axes to obtain $\left(\partial u_{3} / \partial x_{1}\right)$ or $\left(\partial u_{3} / \partial x_{2}\right)$, respectively, where $u_{3}\left(x_{1}, x_{2}\right)$ denotes the out-of-plane surface displacements. As shown in the schematic, the origin of the coordinate system is located at the crack tip of the specimen. The field distribution on the $G_{2}$ plane is spatially filtered by the

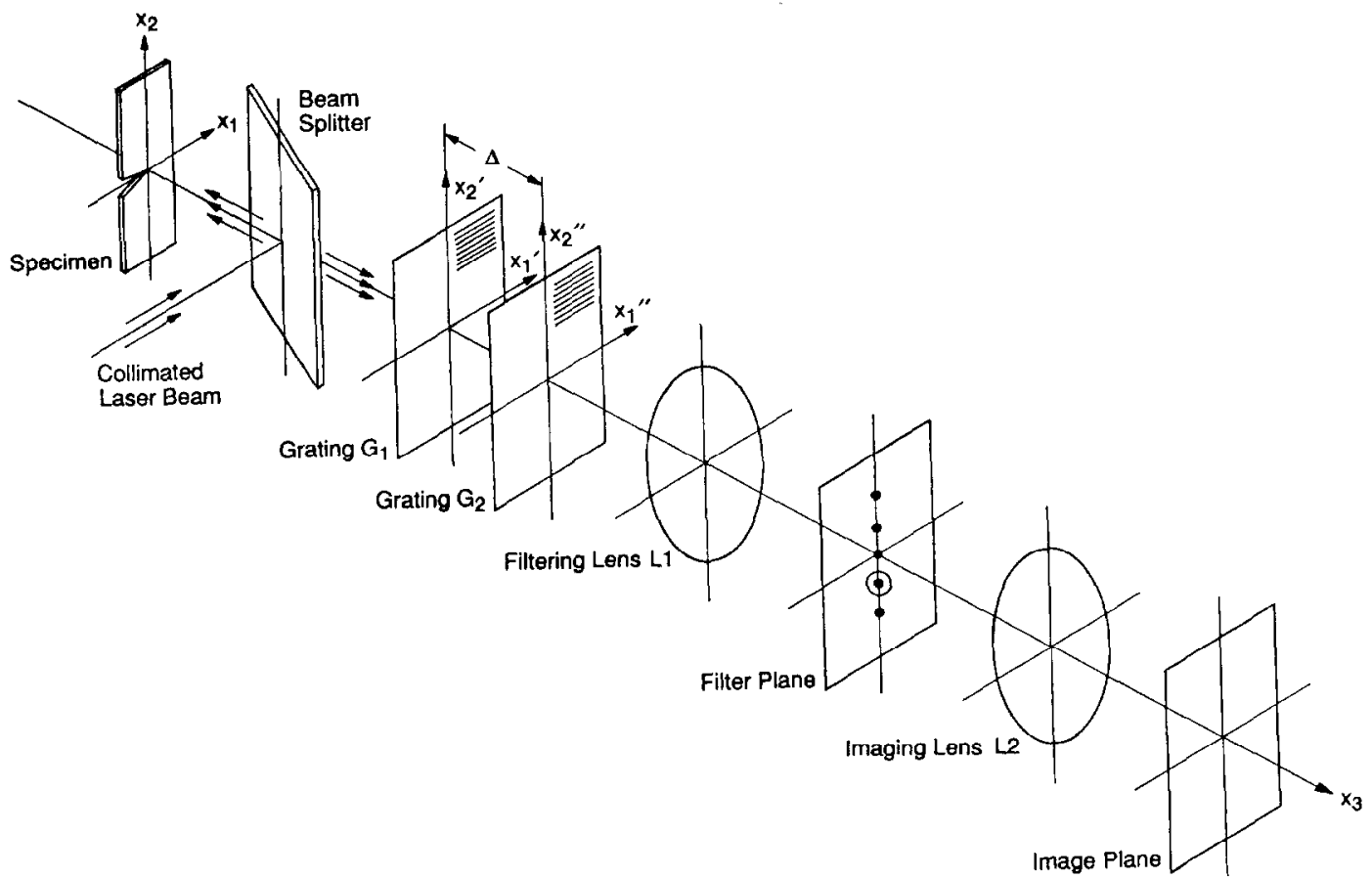

Fig. 1. Schematic of the experimental set-up for CGS. 
filtering lens $L_{1}$ and its frequency content is displayed on its back focal plane. By locating a filtering aperture around either the \pm 1 diffraction orders, the information regarding the displacement gradients is obtained on the image plane of the lens $L_{2}$. In the following sections, a first order diffraction analysis is performed to demonstrate that the information displayed on the image plane indeed corresponds to gradients of the out-of-plane displacement $u_{3}$.

\subsection{Principle}

Figure 2 explains the working principle of the CGS interferometer. For the sake of simplicity, and without losing generality, the line gratings are assumed to have a sinusoidal transmittance. For the case of a plane wave reflected from the flat surface of an undeformed specimen and propagating along the optical axis, the incident wave is diffracted into three plane wave fronts $E_{0}, E_{1}$ and $E_{-1}$ by the first grating $G_{1}$. The magnitude of the angle between the propagation direction of $E_{0}$ and $E_{ \pm 1}$ is given by the grating equation $\theta=\sin ^{-1}(\lambda / p)$, where $\lambda$ is the wave length and $p$ is the grating pitch. Upon incidence on the second grating $G_{2}$, the wave fronts are further diffracted into $E_{0,0}, E_{0,1}, E_{1,-1}, E_{1,0}, E_{1,1}$ etc. These wave fronts which are propagating in distinctly different directions, are then brought to focus at spatially separated diffraction spots on the back focal plane of the filtering lens. The spacing between these diffraction spots is directly proportional to $\sin \theta$ or inversely proportional to the grating pitch $p$.

Now, consider a plane wave normally incident on a deformed specimen surface. The reflected light bundle incident on $G_{1}$ now carries surface displacement gradient information, and is constituted of light rays travelling in arbitrary directions. If a large portion of such a bundle of light has its light rays nearly parallel to the optical axis, each of the diffraction spots will be locally surrounded by a dispersed light field due to the deflected rays. The extent of this dispersion depends on the angle of deflection of the reflected rays. By using a two dimensional aperture at the filtering plane, information existing around one of the spots can be further imaged. This leads to an important but subtle point that should be noted. Since each of the diffraction spots is surrounded by dispersed light containing surface deflection

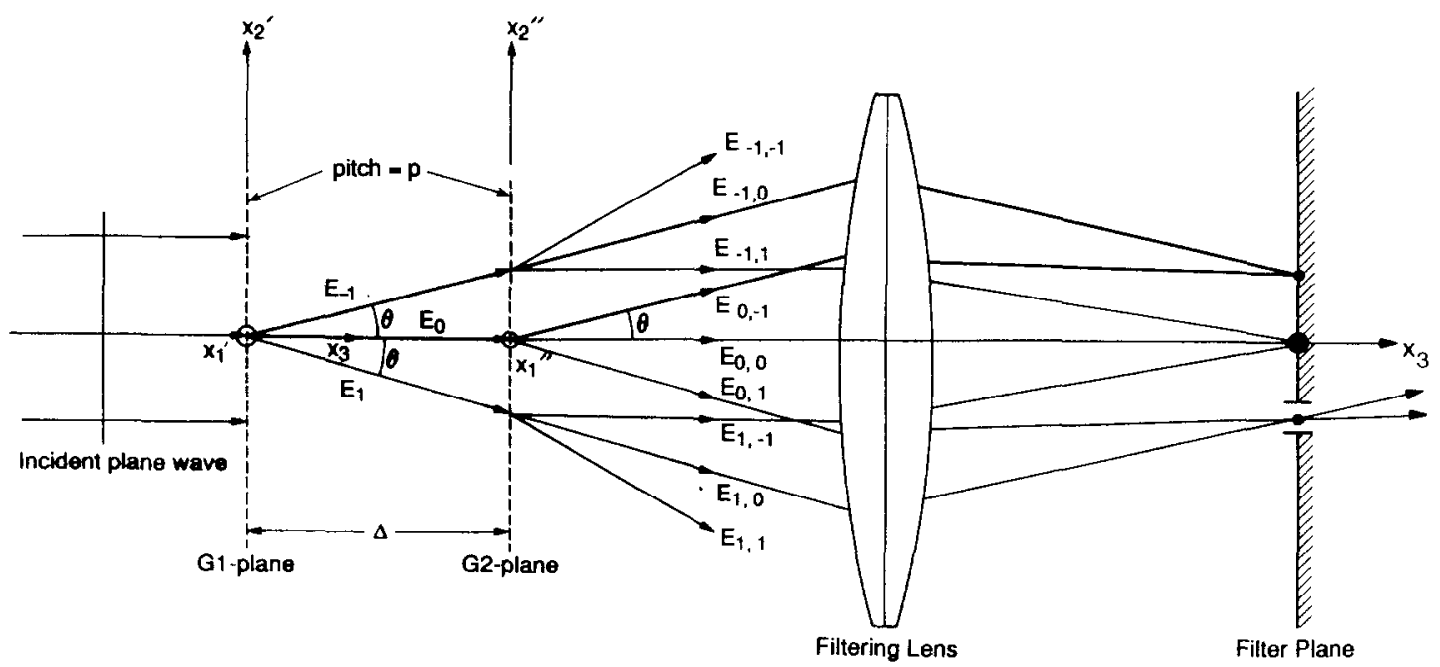

Fig. 2. Working principle for CGS. 
information, overlapping of the information could occur on the filtering plane when the deflection of the ray is sufficiently large (i.e., $\geqslant(\lambda / 2 p)$ ). I Iowever, as will be shown in the following sections, this limitation can easily be overcome by the use of higher density gratings.

\subsection{Analysis}

Consider a specimen whose reflective surface occupies the $\left(x_{1}, x_{2}\right)$ plane in the undeformed state. Upon deformation the reflector surface can be expressed as,

$$
F\left(x_{1}, x_{2}, x_{3}\right)=x_{3}+f\left(x_{1}, x_{2}\right)=0 \text {. }
$$

Consider now, a plane wave which is incident on the specimen along the $-x_{3}$ direction. The unit surface normal $\mathbf{N}$ at a generic point $\mathrm{O}\left(x_{1}, x_{2}\right)$ is given by,

$$
\mathbf{N}=\frac{\nabla F}{|\nabla F|}=\frac{f_{, 1} \mathbf{e}_{1}+f_{, 2} \mathbf{e}_{2}+\mathbf{e}_{3}}{\sqrt{1+f_{, 1}^{2}+f_{, 2}^{2}}},
$$

where $\mathbf{e}_{i}$ denote the unit vectors of the Cartesian coordinate system (see Fig. 3 ) and $f_{, \alpha}$ implies differentiation with respect to $x_{\alpha}$. Let $\mathbf{d}$ be the unit vector along the reflected ray whose direction cosines are $\alpha_{0}, \beta_{0}$ and $\gamma_{0}$. From the law of reflection, the coplanar unit vectors $\mathbf{d}$, $\mathbf{N}$ and $\mathbf{e}_{3}$ are related by $\mathbf{d} \cdot \mathbf{N}=\mathbf{e}_{3} \cdot \mathbf{N}$. This leads to

$$
\mathbf{d}=\left(2 \mathbf{e}_{3} \cdot \mathbf{N}\right) \mathbf{N}-\mathbf{e}_{3} .
$$

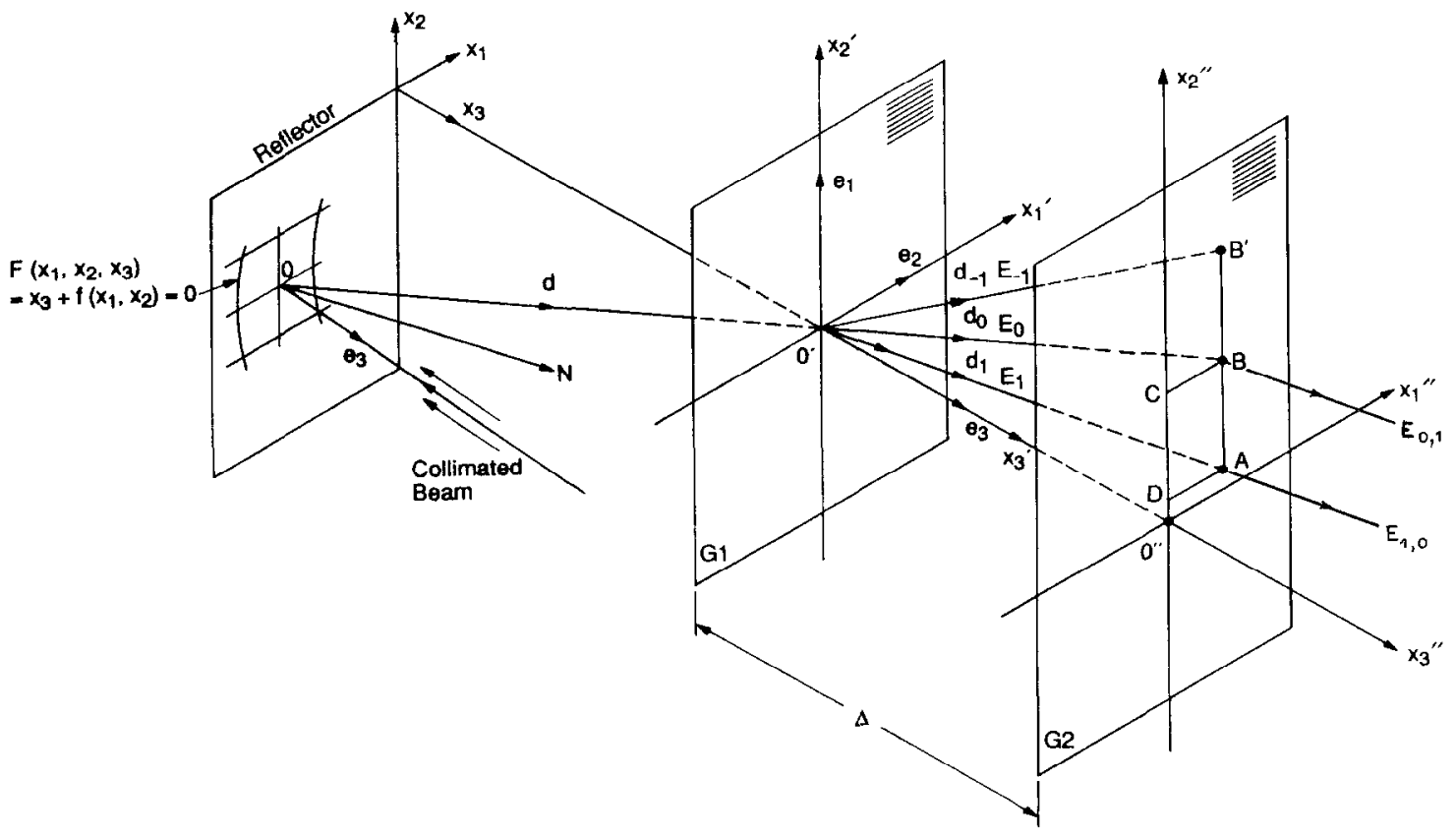

Fig. 3. Diffraction of a generic ray in the CGS. 
By substituting (2) into (3),

$$
\mathbf{d}=\left(\alpha_{0} \mathbf{e}_{1}+\beta_{0} \mathbf{e}_{2}+\gamma_{0} \mathbf{e}_{3}\right)=\frac{2\left(f_{1,} \mathbf{e}_{1}+f_{, 2} \mathbf{e}_{2}+\mathbf{e}_{3}\right)}{\left(1+f_{, 1}^{2}+f_{.2}^{2}\right)}-\mathbf{e}_{3} .
$$

Thus, the direction cosines of $\mathbf{d}$ can be related to the gradients of the function $f$ by

$$
\alpha_{0}=\frac{2 f_{.1}}{\left(1+f_{, 1}^{2}+f_{, 2}^{2}\right)}, \quad \beta_{0}=\frac{2 f_{, 2}}{\left(1+f_{, 1}^{2}+f_{, 2}^{2}\right)}, \quad \gamma_{0}=\frac{\left(1-f_{.1}^{2}-f_{, 2}^{2}\right)}{\left(1+f_{, 1}^{2}+f_{, 2}^{2}\right)} .
$$

The ray along $\mathbf{d}$ upon incidence on the grating $G_{1}$ is split into rays propagating along $\mathbf{d}_{0}$, $\mathbf{d}_{ \pm 1}$ whose amplitudes $E_{0}\left(\mathbf{x}^{\prime}\right), E_{1}\left(\mathbf{x}^{\prime}\right)$ and $E_{-1}\left(\mathbf{x}^{\prime}\right)$ can be represented by,

$$
E_{0}\left(\mathbf{x}^{\prime}\right)=a_{0} \exp \left[\mathrm{i} k \mathbf{d}_{0} \cdot \mathbf{x}^{\prime}\right], \quad E_{1}\left(\mathbf{x}^{\prime}\right)=a_{1} \exp \left[\mathrm{i} k \mathbf{d}_{1} \cdot \mathbf{x}^{\prime}\right], \quad E_{-1}\left(\mathbf{x}^{\prime}\right)=a_{1} \exp \left[\mathrm{i} k \mathbf{d}_{-1} \cdot \mathbf{x}^{\prime}\right],
$$

where $a_{0}$ and $a_{1}$ are constants and the wave number $k=2 \pi / \lambda$. Due to diffraction by the sinusoidal grating $G_{1}$, the propagation directions of the resulting wave fronts are related by,

$$
\mathbf{d}_{ \pm 1}=\boldsymbol{\Omega}_{ \pm 1} \mathbf{d}_{0},
$$

where $\boldsymbol{\Omega}_{ \pm 1}$ are rotation tensors whose matrices of components are given by,

$$
\left[\boldsymbol{\Omega}_{ \pm 1}\right]_{i j}=\left(\begin{array}{ccc}
1 & 0 & 0 \\
0 & \cos \theta & \pm \sin \theta \\
0 & \mp \sin \theta & \cos \theta
\end{array}\right)
$$

and $\theta=\sin ^{-1}(\lambda / p)$. From (7) and (4) we find

$$
\mathbf{d}_{ \pm 1}=\left[\alpha_{0} \mathbf{e}_{1}+\left(\beta_{0} \cos \theta \pm \gamma_{0} \sin \theta\right) \mathbf{e}_{2}+\left(\gamma_{0} \cos \theta \mp \beta_{0} \sin \theta\right) \mathbf{e}_{3}\right]
$$

Now, with reference to Fig. 3, on plane $G_{2}$ we have

$$
E_{0}\left(\boldsymbol{O}^{\prime} \boldsymbol{B}\right)=a_{0} \exp \left[\mathrm{i} k \mathbf{d}_{0} \cdot \boldsymbol{O}^{\prime} \boldsymbol{B}\right]
$$

In addition, since $\left|\boldsymbol{O}^{\prime} \boldsymbol{B}\right| \mathbf{d}_{0} \cdot \mathbf{c}_{3}=\left|\boldsymbol{O}^{\prime} \boldsymbol{B}\right| \gamma_{0}=\Delta$, one finds that $\left|\boldsymbol{O}^{\prime} \boldsymbol{B}\right|=\left(\Delta / \gamma_{0}\right)$. Hence,

$$
E_{0}\left(\boldsymbol{O}^{\prime} \boldsymbol{B}\right)=a_{0} \exp \left[\mathrm{i} k\left(\frac{\Delta}{\gamma_{0}}\right)\right]
$$

In like manner,

$$
\begin{aligned}
\left|\boldsymbol{O}^{\prime} \boldsymbol{A}\right| \mathbf{d}_{1} \cdot \mathbf{e}_{3} & =\left|\boldsymbol{O}^{\prime} \boldsymbol{A}\right|\left(\gamma_{0} \cos \theta-\beta_{0} \sin \theta\right)=\Delta \\
\left|\boldsymbol{O}^{\prime} \boldsymbol{B}^{\prime}\right| \mathbf{d}_{-1} \cdot \mathbf{e}_{3} & =\left|\boldsymbol{O}^{\prime} \boldsymbol{B}^{\prime}\right|\left(\gamma_{0} \cos \theta+\beta_{0} \sin \theta\right)=\Delta
\end{aligned}
$$


and thus,

$$
\begin{aligned}
& E_{1}\left(\boldsymbol{O}^{\prime} \boldsymbol{A}\right)=a_{1} \exp \left[\mathrm{i} k \mathbf{d}_{1} \cdot \boldsymbol{O}^{\prime} \boldsymbol{A}\right]=a_{1} \exp \left[\mathrm{i} k \frac{\Delta}{\left(\gamma_{0} \cos \theta \beta_{0} \sin \theta\right)}\right] \\
& E_{-1}\left(\boldsymbol{O}^{\prime} \boldsymbol{B}^{\prime}\right)=a_{1} \exp \left[i k \mathbf{d}_{-1} \cdot \boldsymbol{O}^{\prime} \boldsymbol{B}^{\prime}\right]=a_{1} \exp \left[i k \frac{\Delta}{\left(\gamma_{0} \cos \theta+\beta_{0} \sin \theta\right)}\right]
\end{aligned}
$$

\subsection{Spatial filtering}

The wave fronts $E_{0}, e_{ \pm 1}$ will undergo further diffraction upon incidence on $G_{2}$ into secondary wave fronts $E_{(0,0)}, E_{(0,1)}, E_{(1,-1)}^{\prime}, E_{(1,0)}, E_{(1,1)}^{\prime}$ etc. Of these secondary diffractions, $E_{(0,1)}$ and $E_{(1,0)}$ have their propagation direction along $\mathbf{d}_{1}, E_{(0,1)}$ and $E_{(-1,0)}$ along $\mathbf{d}_{-1}$ and $E_{(0,0)}, E_{(-1,1)}$ and $E_{(1,-1)}$ along $\mathbf{d}_{0}$, Fig. 3. If information is spatially filtered by blocking all but \pm 1 diffraction order, only the wave fronts $E_{(0, \pm 1)}$ and $E_{( \pm 1,0)}$ contribute to the formation of the image. Noting that the two wave fronts do not acquire any additional relative phase differences after $G_{2}$, the amplitude distribution on the image plane is,

$$
E_{\mathrm{im}}=\left.\left(E_{0}+E_{ \pm 1}\right)\right|_{x_{3}^{\prime}=\Delta}=a_{0} \exp \left[\mathrm{i} k\left(\frac{\Delta}{\gamma_{0}}\right)\right]+a_{1} \exp \left[\mathrm{i} k \frac{\Delta}{\left.\gamma_{0} \cos \theta \mp \beta_{0} \sin \theta\right)}\right] \text {. }
$$

Hence, the intensity distribution on the image plane is,

$$
I_{\mathrm{im}}=E_{\mathrm{im}} E_{\mathrm{im}}^{*}=a_{0}^{2}+a_{\mathrm{1}}^{2}+2 a_{0} a_{1} \cos \left\{k \Delta\left[\frac{\gamma_{0}(\cos \theta-1) \mp \beta_{0} \sin \theta}{\gamma_{0}\left(\gamma_{0} \cos \theta \mp \beta_{0} \sin \theta\right)}\right]\right\},
$$

where $E_{\mathrm{im}}^{*}$ is the complex conjugate of $E_{\mathrm{im}}$. Under small $\theta$ approximation, the above equation simplifies to

$$
I_{\mathrm{im}}=a_{0}^{2}+a_{1}^{2}+2 a_{0} a_{1} \cos \left(\frac{k \Delta \beta_{0} \theta}{\gamma_{0}^{2}}\right) .
$$

Thus, $I_{\mathrm{im}}$ denotes an intensity variation on the image plane whose maxima occur when

$$
\frac{k \Delta \beta_{0} \theta}{\gamma_{0}^{2}}=2 n \pi, \quad n=0, \pm 1, \pm 2, \ldots
$$

where $n$ denotes fringe orders.

From (5) the direction cosines $\alpha_{0}, \beta_{0}$ and $\gamma_{0}$ are related to the gradients of the function $f$. Then, the above equation can be written as,

$$
k \Delta \theta\left[2 f_{, 2}\left(\frac{1+|\nabla f|^{2}}{\left(1-|\nabla f|^{2}\right)^{2}}\right)\right]=2 n \pi
$$


When $|\nabla f|^{2} \ll 1,(20)$ becomes,

$$
f_{, 2} \approx\left(\frac{n p}{2 \Delta}\right), \quad n=0, \pm 1, \pm 2, \ldots
$$

where the fact that $\theta \approx(\lambda / p)$ and $k=2 \pi / \lambda$ are made use of. Similarly, when the gratings are oriented with their principal direction coinciding with the $x_{1}$-axis,

$$
f_{, 1} \approx\left(\frac{m p}{2 \Lambda}\right), \quad m=0, \pm 1, \pm 2, \ldots
$$

Thus, (21) and (22) are the governing equations for the method of CGS. It should be noted that these equations are similar in form to those of moiré deflectometry [8] and reflection moiré methods [9] based on geometric optics. It is clear from the above two equations that the sensitivity of the method could be increased by either increasing the grating separation distance $\Delta$ or decreasing the grating pitch $p$.

\section{Experimental results}

Two experiments are performed to demonstrate the applicability of CGS in solid mechanics in general and fracture mechanics in particular. First, the gradients of a known function $f$ were used to test the reliability of the measurements. For this purpose, a spherical wave front was generated using a convex lens of focal length $f_{l}=546 \mathrm{~mm}$. Line gratings of density 40 lines per $\mathrm{mm}$ were oriented with their principal direction coinciding with the $x_{2}$-axis and were separated by a distance of $\Delta=22 \mathrm{~mm}$ to produce fringes that are shown in Fig. 4 . The fringe spacing $\left(x_{2} / n\right)$ corresponding to the gradients in the $x_{2}$-direction namely, $f_{, 2}$ measured from the fringe pattern is $0.66 \mathrm{~mm} /$ fringe. The spherical wave front emerging from the convex lens can be described by,

$$
f\left(x_{1}, x_{2}\right)=\frac{x_{1}^{2}+x_{2}^{2}}{2 f_{l}}
$$

and hence (in transmission),

$$
\beta_{0}=f_{, 2}=\left(\frac{x_{2}}{f_{l}}\right)=\frac{n p}{\Delta},
$$

which corresponds to straight line fringes as shown in the figure. For the experimental parameters used in this demonstration, the fringe density expected from the above equations is $0.63 \mathrm{~mm} /$ fringe which is in good agreement with the experimental measurements.

Secondly, CGS was used to obtain gradients of the out-of-plane displacement $u_{3}\left(x_{1}, x_{2}\right)$ $\left(=-f\left(x_{1}, x_{2}\right)\right)$ around a deformed crack tip in a three point bend fracture specimen made of AISI 4340 steel. The dimensions of the specimen and the experimental set-up are shown 


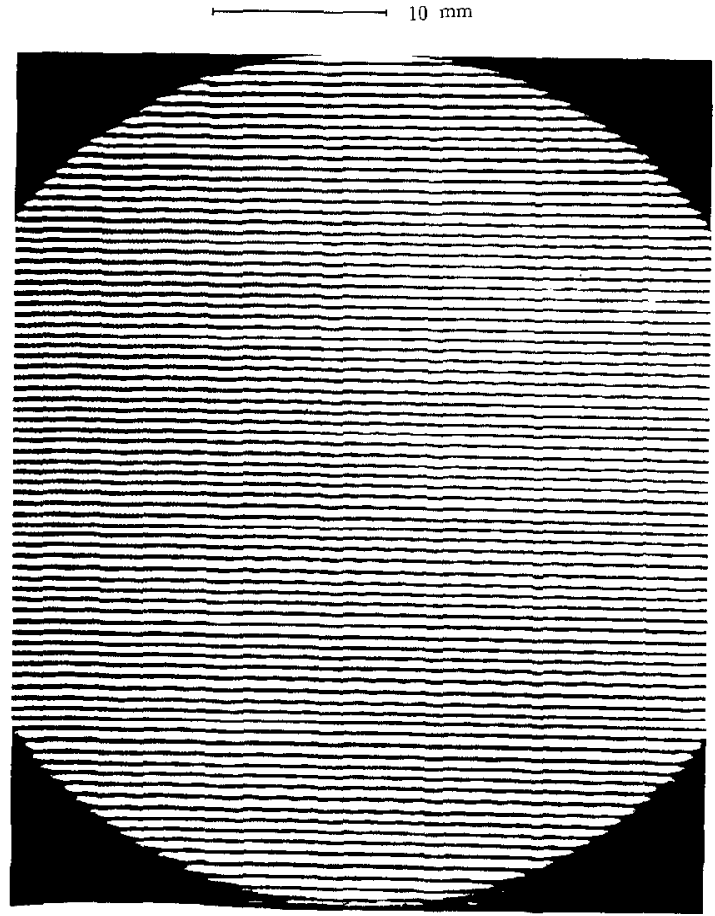

Fig. 4. Contours of constant $f_{, 2}$ for a spherical wave front.

in Figs. 5 and 6. The specimen has an electro-discharge machined notch which is $25 \mu \mathrm{m}$ wide and $30 \mathrm{~mm}$ deep through a $10 \mathrm{~mm}$ thick plate. The specimen was heat treated to have a yield stress of $1350 \mathrm{MPa}$. One of the surfaces of the specimen was lapped and polished to obtain a flat reflective test surface. The specimen was statically loaded in a hydraulic loading frame in displacement control mode. The load and the load point displacements were measured during the test. The optical set-up has two Ronchi rulings of 40 lines per $\mathrm{mm}$ density and are separated by a distance $(\Delta)$ of $21 \mathrm{~mm}$ giving a sensitivity of measurement $6.05 \times 10^{-4} \mathrm{rad} /$ fringe. In Fig. 7 fringe patterns representing contours of constant $\left(\partial u_{3} / \partial x_{1}\right)$ and $\left(\partial u_{3} / \partial x_{2}\right)$ corresponding to three load levels $P / P_{0}=0.38,0.61,0.71$ are shown where $P_{0}$ is the plane stress limit load for the specimen. For the lowest load level of the three

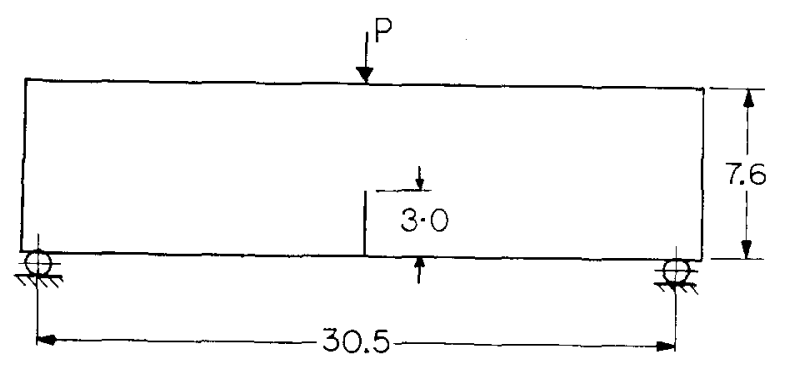

Thickness, $h=1.0$

Fig. 5. Specimen geometry or the 3-point bend fracture specimen. 


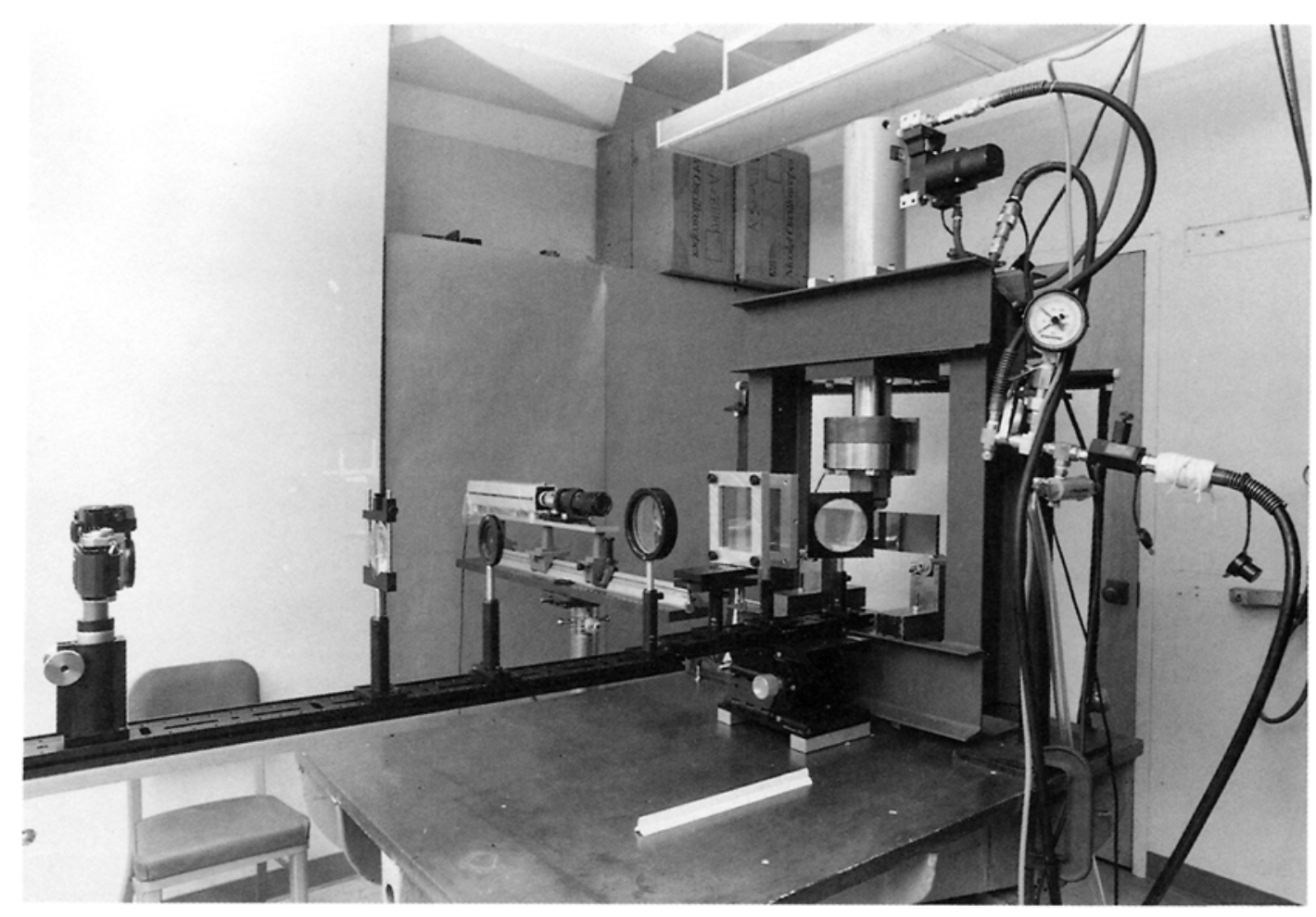

Fig. 6. Experimental arrangement for fracture experiment using CGS.

patterns shown (Fig. 7a), the near tip out-of-plane displacement field was assumed to be given by the lincar clastic, asymptotic, plane stress expression,

$$
\begin{aligned}
f(r, \phi) & =-u_{3}(r, \phi)=\frac{v h}{2 E}\left[\sigma_{11}(r, \phi)+\sigma_{22}(r, \phi)\right] \\
& \approx \frac{v h K_{\mathrm{I}}}{E \sqrt{2 \pi r}} \cos (\phi / 2)+\left(\frac{v h}{2 E}\right) \sigma_{11}^{0}+o(1), \text { as } r \rightarrow 0,
\end{aligned}
$$

where $r=\sqrt{x_{1}^{2}+x_{2}^{2}}, \phi=\tan ^{-1}\left(x_{2} / x_{1}\right), h$ is the specimen thickness, $E$ is Young's modulus, $v$ is Poisson's ratio, $\sigma_{11}^{0}$ is the constant term in the asymptotic expansion for the stresses, and $K_{\mathrm{I}}$ is the mode I stress intensity factor to be determined by CGS.

By using (21) and (22), the stress intensity factor can now be obtained from the fringe pattern, Fig. 7a, through:

$$
\left(K_{1}\right)_{\mathrm{CGS}}=\frac{2 E \sqrt{2 \pi} r^{3 / 2}}{v h \cos (3 \phi / 2)} \cdot\left(\frac{m p}{2 \Delta}\right), \quad m=0, \pm 1, \pm 2, \ldots
$$

In the above expression, $m$ is the fringe order and $\left(K_{1}\right)_{\text {CGS }}$ denotes the experimentally obtained value for the stress intensity factor. Note that the constant term $\sigma_{11}^{0}$ does not appear 

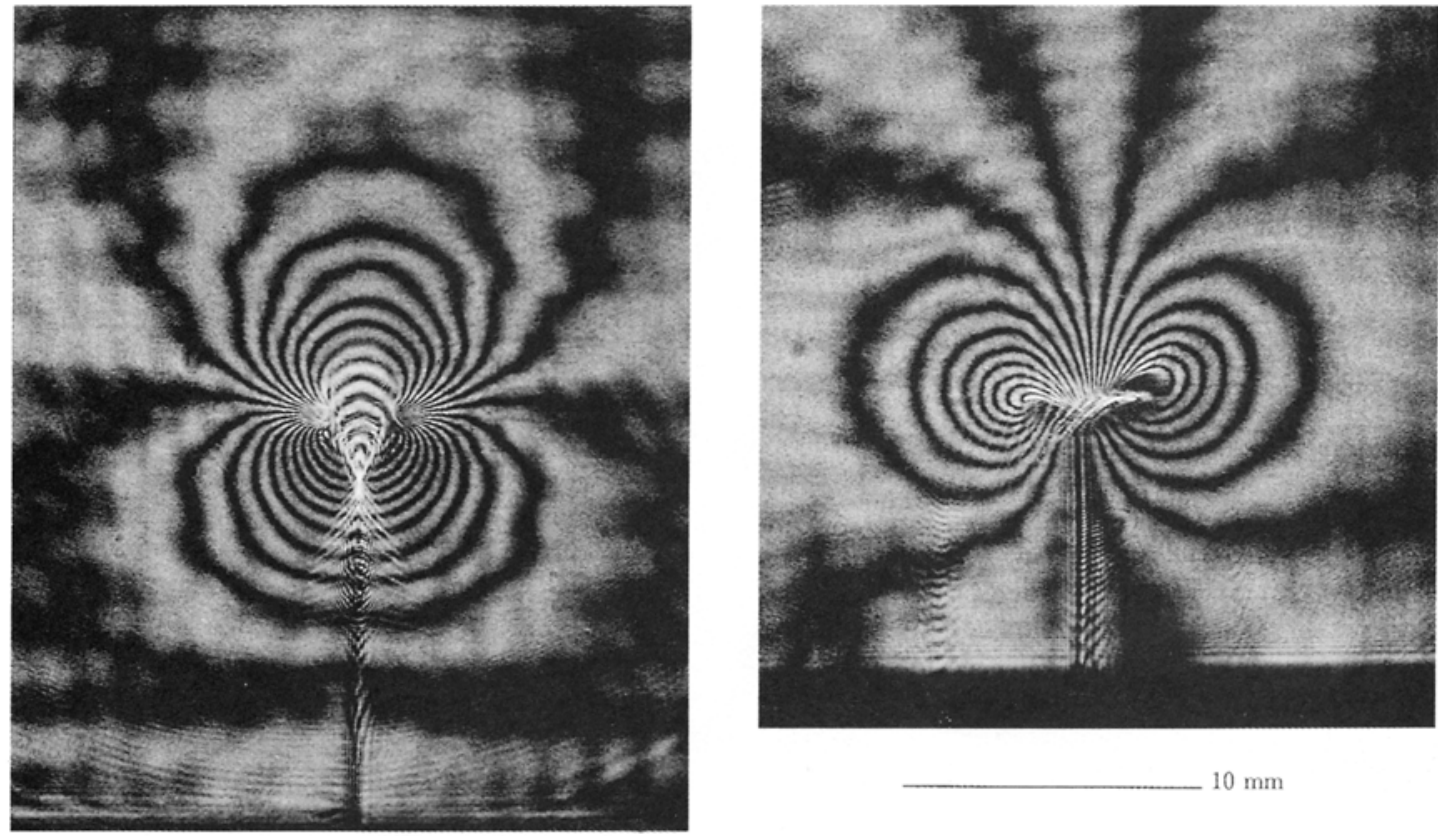

$10 \mathrm{~mm}$

(a)
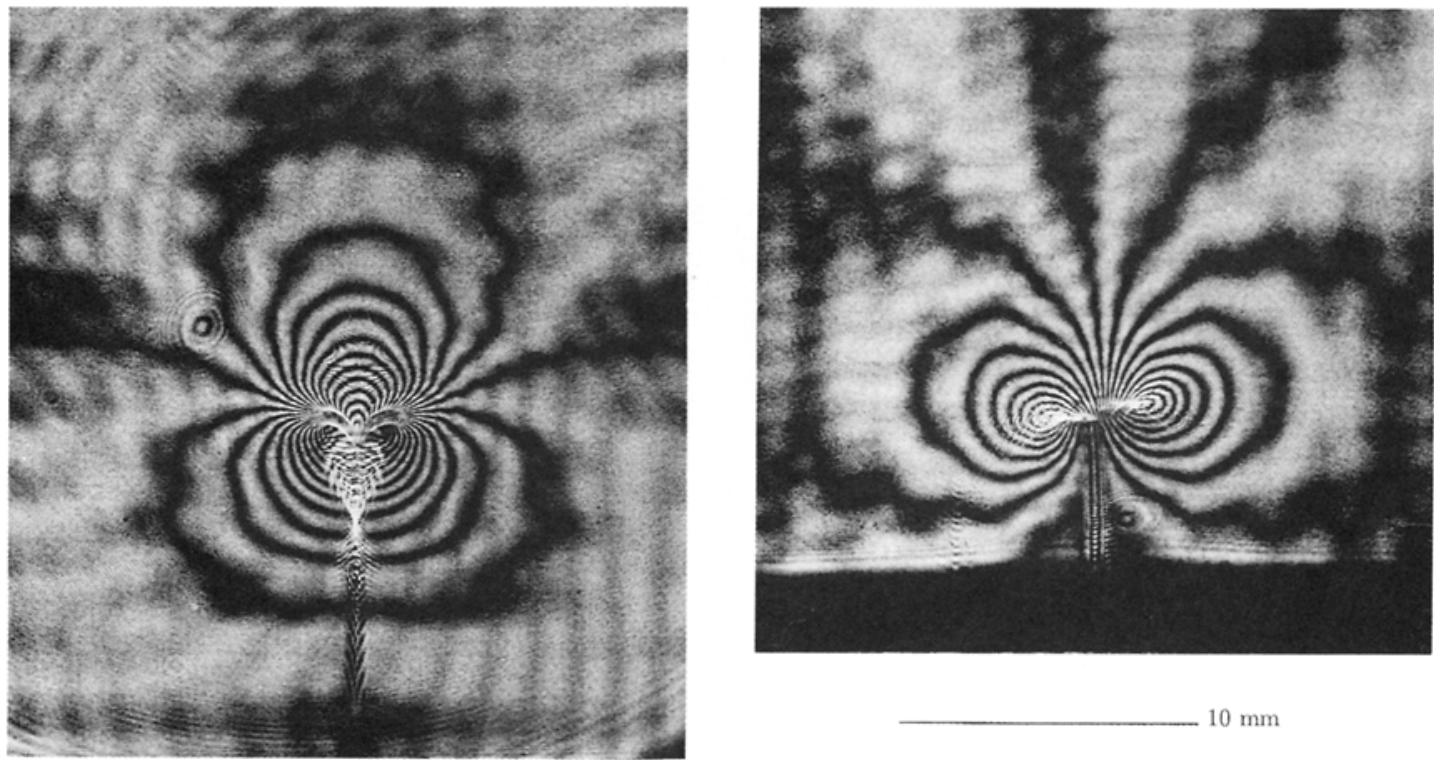

$10 \mathrm{~mm}$

(b)

Fig. 7. (a) Contours of $\left(\partial u_{3} / \partial x_{1}\right)$ and $\left(\partial u_{3} / \partial x_{2}\right)$ for $\left(P / P_{0}\right)=0.38$. (b) Contours of $\left(\partial u_{3} / \partial x_{1}\right)$ and $\left(\partial u_{3} / \partial x_{2}\right)$ for $\left(P / P_{0}\right)=0.61$.

in (26) because the measurements are sensitive to surface gradients only. $\left(K_{\mathrm{I}}\right)_{\mathrm{CGS}}$ can now be computed from different fringes corresponding to different $(r, \phi)$ pairs. If the out-of-plane displacement field of the specimen surface is indeed given by (25), the experimentally obtained $\left(K_{\mathrm{I}}\right)_{\mathrm{CGS}}$ should be independent of the location of measurement (choice of $r, \phi$ pairs in (26)). In such a case, $\left(K_{\mathrm{I}}\right)_{\mathrm{CGS}}$ should agree with the stress intensity factor $\left(K_{\mathrm{I}}\right)_{2 \mathrm{D}}$ obtaincd from boundary value measurements by means of a two dimensional analysis [10]. 

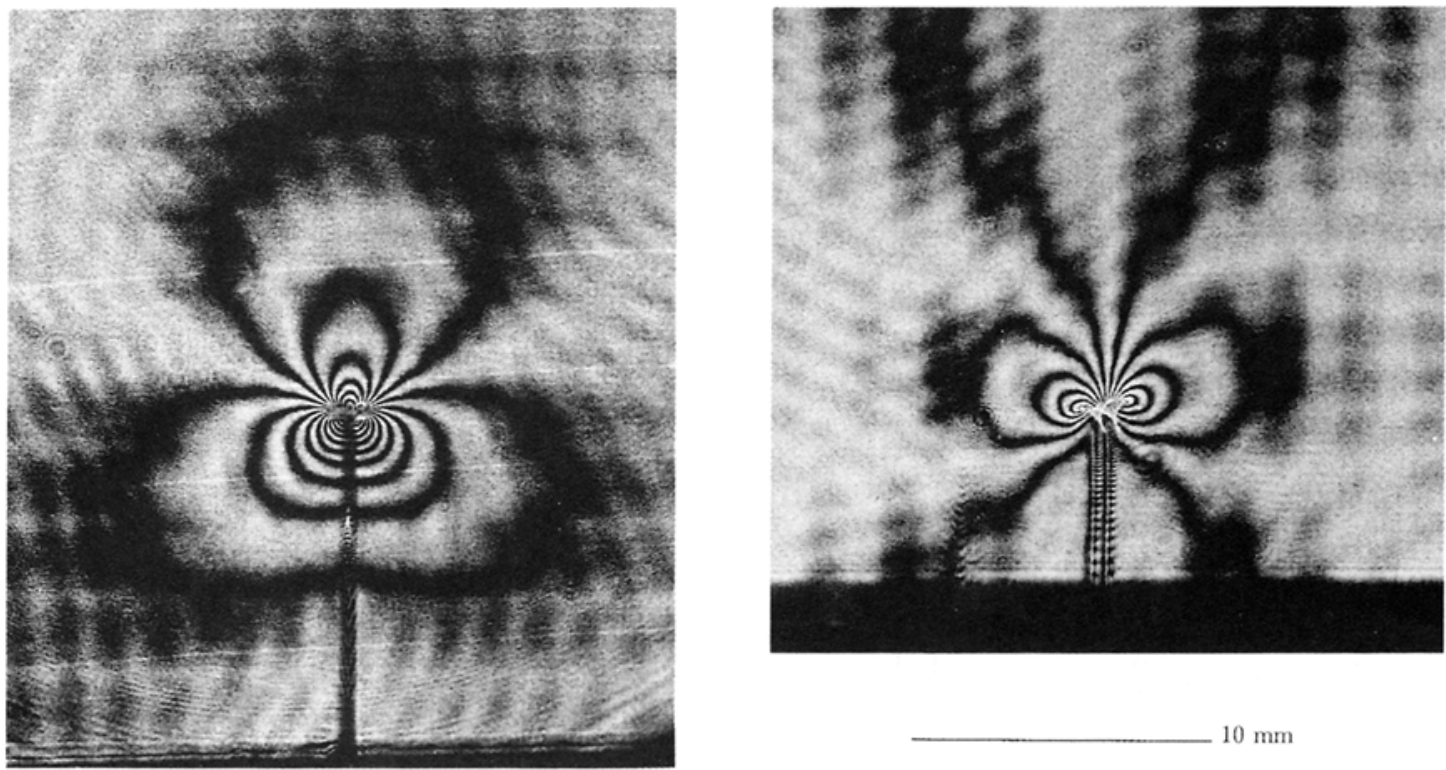

$10 \mathrm{~mm}$

(c)

Fig. 7. (c) Contours of $\left(\partial u_{3} / \partial x_{1}\right)$ and $\left(\partial u_{3} / \partial x_{2}\right)$ for $\left(P / P_{0}\right)=0.71$.

However, near the crack tip three dimensional effects are expected to violate the plane stress assumption which leads to (25). Indeed, the experimental results reported by Rosakis and Ravi-Chandar [12] and supported by the analytical investigations of Yang and Freund [11] have shown that (25) is a good description of the surface out-of-plane displacements only for radial distances greater than $0.5 h$. This behaviour is also evident from the current experimental results displayed in Fig. 8. Here, $\left(K_{\mathrm{I}}\right)_{2 \mathrm{D}}$ is obtained through the boundary load and the results compiled in [10]. $\left(K_{1}\right)_{\text {cos }}$ is obtained from fringes at different radial distances along the $\phi=0$ line by using (26). It is clear from the figure that the measured value of

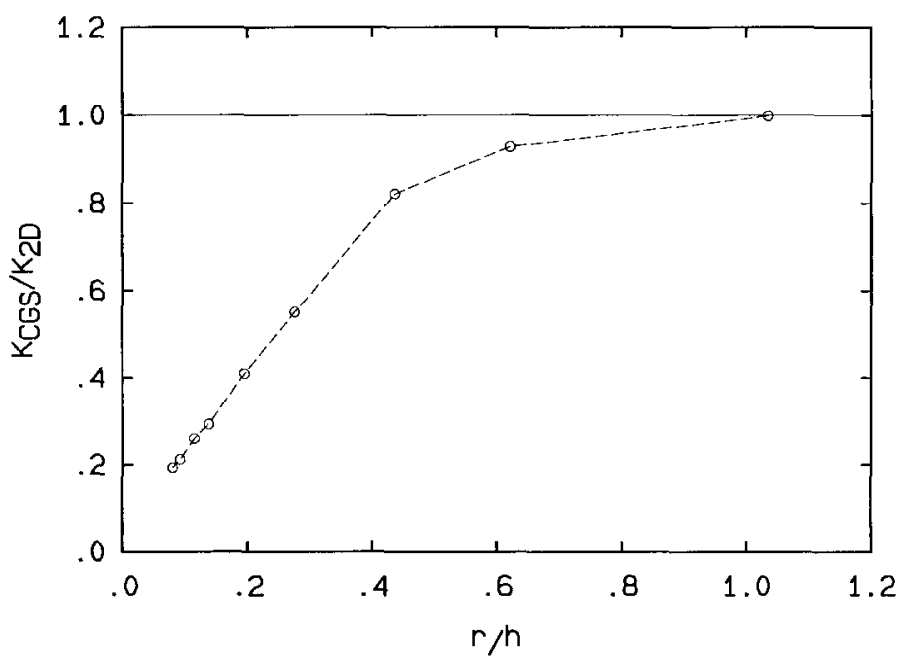

Fig. 8. Experimental results from $\left(\partial u_{3} / \partial x_{1}\right)$ fringes along $(r, \phi=0)$ line for $\left(P / P_{0}\right)=0.38$. 
$\left(K_{\mathrm{I}}\right)_{\mathrm{CGS}}$ is in good agreement with $\left(K_{\mathrm{I}}\right)_{2 \mathrm{D}}$ for $(r / h)>0.5$. However, for $(r / h)<0.5$, a marked underestimation of the inferred stress intensity factor is obscrved. This is consistent with the results shown in Figs. 5 and 6 of [12] which are obtained by using the optical method of caustics. This is to be expected since both caustics and CGS rely on surface out-of-plane displacement gradients.

The fringe patterns in Figs. $7 b, c$ correspond to substantial near tip plastic deformations and as such the use of (25) and (26) is inappropriate. Direct comparison of the experimentally obtained displacement fields with a three dimensional elastic-plastic finite element analysis of the specimen is underway.

\section{Conclusions}

This paper provides a detailed diffraction analysis of the proposed interferometer, Coherent Gradient Sensor. The analysis indicates that the resulting interference fringes represent contours of constant surface gradients. Although the method bears similarities in its operating principle with moiré deflectometry [8], it has additional advantages because it uses coherent optics. Unlike moiré deflectometry, which is limited by diffraction effects, this method utilizes such effects by making use of high density gratings and spatial filtering procedure. Also, spatial filtering enhances the contrast and sharpness of the fringes.

The feasibility of CGS as a means of quantifying crack tip deformation fields has been demonstrated in the elastic and plastic regimes. Simplicity of the optical set-up, sharp and high contrast fringes and limited loss of light intensity make CGS a suitable candidate for dynamic crack initiation and propagation studies. Its relative insensitivity to laboratory vibrations comes as an additional advantage.

\section{Acknowledgements}

The authors wish to acknowledge the support of the work by the Office of the Naval Research through grant N00014-85-K-0596.

\section{References}

1. J.W. Dally, in Optical Methods in Mechanics of Solids, A. Lagarde (ed.), Sijthoff and Noordoff (1980).

2. B.S.J. Kang, A.S. Kobayashi and D. Post, Experimental Mechanics 3 (1987) 234-245.

3. F.P. Chiang and T.V. Hareesh, International Journal of Fracture 36 (1988) 243-257.

4. A.J. Rosakis, A.T. Zehnder and R. Narasimhan, Optical Engineering 27, No. 8 (1988) 596-610.

5. J. Beinert and J.F. Kalthoff, in Mechanics of Fracture, Vol. VII, G. Sih (ed.), Sijthoff and Noordoff (1981) 281-320.

6. A.J. Rosakis, C.C. Ma and L.B. Freund, Journal of Applied Mechanics 105 (1983) 777-782.

7. O. Kafri, Optics Letters 5 (1980) 555-557.

8. I. Glatt and O. Kafri, Optics and Lasers in Engineering 8 (1988) 277-320.

9. F.P. Chiang, in Manual of Stress Analysis Techniques, A.S. Kobayashi (ed.), SESA, 3rd ed. (1978).

10. D.P. Rooke and D.P. Cartwright, Compendium of Siress Intensity Faciors, Her Majesty's Stationery Office (1975).

11. W. Yang and L.B. Freund, International Journal of Solids and Structures 21, No. 9 (1985) 977-994.

12. A.J. Rosakis and K. Ravi-Chandar, International Journal of Solids and Structures, 22 (1986) 121-138. 